Research Article

\title{
Compensation for Cross-Coupled Dynamics of Dual Twisted-String Actuation Systems
}

\author{
Lei Jiang, ${ }^{1}$ Yuejuan Li, ${ }^{2}$ and Marvin H. Cheng ${ }^{1}$ \\ ${ }^{1}$ Department of Mechanical and Aerospace Engineering, West Virginia University, Morgantown, WV 26506, USA \\ ${ }^{2}$ Beijing University of Technology, Beijing 100124, China \\ Correspondence should be addressed to Lei Jiang; lejiang@mix.wvu.edu
}

Received 8 December 2015; Accepted 25 February 2016

Academic Editor: Petko Petkov

Copyright (c) 2016 Lei Jiang et al. This is an open access article distributed under the Creative Commons Attribution License, which permits unrestricted use, distribution, and reproduction in any medium, provided the original work is properly cited.

\begin{abstract}
Twisted-string actuation devices have been adopted in various robotic systems due to their advantages of compact size and simple structure. To precisely control the displacement of such devices, a dual-direction actuating mechanism, which provides both extension and contraction of two strings simultaneously, must be implemented. Due to the physical properties of twisted string, the actuator has problems of nonlinear length variation and cross-coupled relationships between two strings. In this study, two controllers (PID-FC and LQR-FC) were synthesized with the consideration of cross-coupling dynamics between the two axes. The experimental results demonstrate the performance of both tracking and synchronization responses of these two types of controllers.
\end{abstract}

\section{Introduction}

Force transmission with cable/tendons has been widely adopted in various industrial applications. The low inertia and high precision of cable transmission also bring many advantages in different mechanical systems. Among different types of force transmission methods, the twistedstring actuation system has gained significant attention [14] in the past few years, and it has been proven to be an effective means to provide linear movement as well. Its unique properties of flexibility and compact size make such an actuation system a great device to advance/retract objects from a distance. Twisted-string actuation has been applied to different applications, including robotic devices $[5,6]$ and small lifting mechanisms. However, most current studies only focus on the linear motion actuation provided by a single actuator. It can be an issue if the desired trajectory of an object is bidirectional. To advance and retract an object, force must be applied on both sides. Thus, unless forces on both moving directions can be available, it cannot always retract/extend the cable back to its original position precisely. With this consideration, the control of linear motion implemented by twisted-string actuation devices must be a dual-axial motion system. In other words, a pair of twisted-string actuators is necessary to extend/retract the movement simultaneously.
The synchronization of multiaxis movement can be compensated by either mechanical linkages or software compensation. Various controllers that compensate for the synchronization of multiaxial systems, such as master-slave framework, equal-status method, and feedback of crosscoupling dynamics, have been proposed in the past [7] by different research groups. For instance, a master-slave control method has been investigated for applications of motion synchronization control [8]. Such kind of controllers has the benefit of reducing contour error of multiaxial motion systems, in which the motion characteristics of individual axes are different. Tsujiuchi et al. [9] have developed a pneumatic robot hand with a master-slave controller to follow the trajectories of the user movements. In 1980, Koren [10] introduced a cross-coupled controller for dual-axis feed driver systems to improve the synchronization performance by adopting the equal-status viewpoint. With the consideration of the coupling dynamics among different axes, different approaches were investigated to compensate both tracking and synchronization errors in different mechanical systems.

A geometrical framework for coordination control of multiple motion axes using three-dimensional curves has been formulated by Chiu and Tomizuka [11]. With the framework, various control strategies for multiaxis control systems 


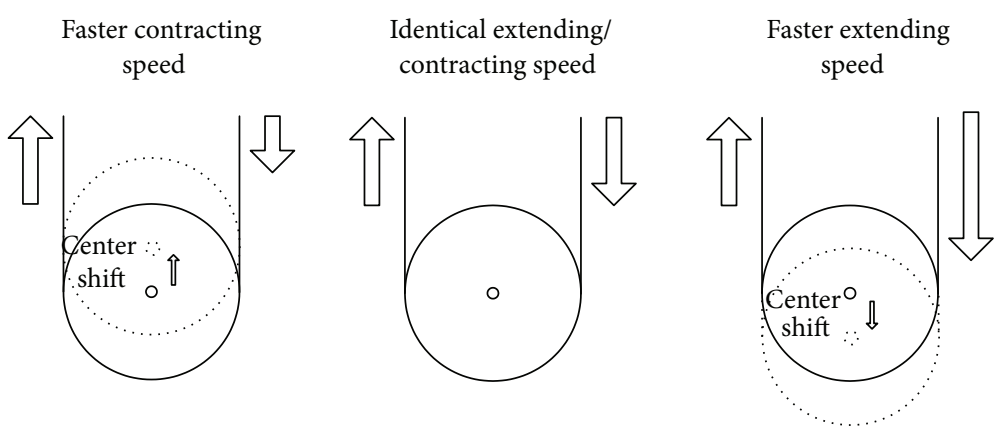

FIGURE 1: Three scenarios of synchronization between extending and contracting strings.

have also begun to be investigated since then. Jeong and You [12] adopted a cascaded structure to control both acceleration and position. With this structure, the robustness of speed of each axis against disturbances and synchronization errors is guaranteed. A framework of adaptive control laws using a backstepping procedure has been proposed by Cheng et al. [13-15] to control a multiaxis motion system. With their proposed LQ and adaptive controllers, both tracking and synchronization performance can be achieved.

In this study, a dual twisted-string actuation system was adopted to drive a pulley used as a joint in a robotic system. The two twisted-string actuators are used to drive the joint in the extension and flexion directions. As the strings start to be twisted or untwisted, the joint starts to rotate. Figure 1 demonstrates the configuration of movements created by the extension and contraction of the strings. The dual twisted-string actuation system has the advantages of compact size and flexible actuation range. Another advantage of this type of actuation device is that the driving motors do not need to be attached to the joint if available space is limited. However, twisted-string actuation can suffer from nonlinear length variation, hysteresis, and synchronization issues. In particular, the linear displacements of the two strings are cross-coupled with each other. Unsynchronized motion can make the joint be slack. To compensate for the movement, two different control methods, PID and LQR with cross-coupling dynamics, were applied to the twistedstring actuators to compensate for both tracking and synchronization responses. However, though many multiaxial controllers can provide adequate tracking and synchronization performance, disturbance rejection was still not well considered. In particular, if a twisted-string actuation needs to drive the motion of a robotic system, disturbance rejection can be an important issue that needs to be addressed. This paper investigates the controller synthesis that compensates for both tracking and synchronization of a dual twisted-string actuation system with the consideration of cross-coupling dynamics and disturbance rejection.

This paper is organized as follows. In Section 2, the mathematical models of the twisted-string system and driving actuators are identified. The experimental setup and the corresponding linear range are introduced as well. The phenomenon of synchronization of the dual-axis system is discussed in Section 3. In Section 4, PID and LQ controllers are synthesized with the consideration of cross-coupling

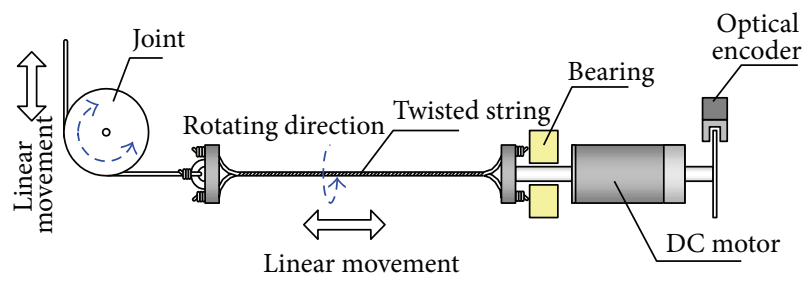

FIGURE 2: Configuration for a twisted-string actuation device.

dynamics. The tracking and synchronization performance of both controllers are compared in this section. Conclusions are addressed in Section 5.

\section{Setup and Modeling of Twisted-String System}

2.1. Modeling of Twisted-String Actuation. The twisted-string actuator converts rotational movement of a motor into linear movement by a string as shown in Figure 2. With one end fixed on a DC motor shaft and the other end fixed on the load, two or multiple strands can be twisted by the rotational movement of the motor. As the motor starts to rotate, the string connected to the motor shaft starts to reduce the distance between both ends and generate a linear motion. The original length of a single strand is $L_{0}$. Once the driven motor starts to twist the strands, the lengths of individual strands become $L$, which also changes the length of the string $d$. A mathematical model for such a twisted-string actuator has been developed by Würtz et al. [1,2] to estimate the length of the twisted string. The relationship between the angle $\alpha$, the revolution of motor shaft $\theta$, and the length $L$ of a single strand can be concluded from the geometric configuration of the helix formed by the strands. They are

$$
\begin{aligned}
L & =\sqrt{\theta^{2} r^{2}+d^{2}}, \\
\sin \alpha & =\frac{\theta \cdot r}{L}, \\
\cos \alpha & =\frac{d}{L}, \\
\tan \alpha & =\frac{\theta \cdot r}{d},
\end{aligned}
$$



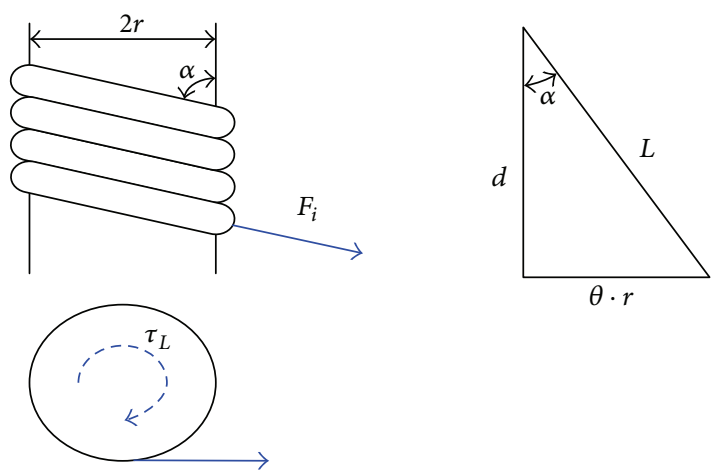

Figure 3: Geometric configuration of a single twisted strand.

where $\alpha$ is the angle between the strand and the axis of twist, $L$ is the length of a single strand as it is unwrapped, $\theta$ is the total revolution of motor shaft, and $d$ is the nominal length of the string after being twisted. The geometric configuration of the twisted string is shown in Figure 3. The coefficient $r$ is the effective radius of the string, which is a combination of multiple strands. The effective $r$ is the distance between the outer strands and center of rotation and is given by $r=r_{s}+r_{c}$, where $r_{s}$ is the radius of the central neutral strand and $r_{c}$ is the radius of the surrounding outer or effective strands. The twisted strand is assumed to be a linear spring which only provides tensile force. As the strands start to be twisted, the length of a single strand is changed from its original, or untwisted, length $L_{0}$ to $L$. Then the relationship between the force and the changed length of the strand can be derived from Hooke's law, which is

$$
F_{i}=K\left(L-L_{0}\right)=K\left(\sqrt{r^{2} \theta^{2}+d^{2}}-L_{0}\right),
$$

where $K$ is the stiffness coefficient of the strands. Thus, the total length of a strand $L$ is a function of the tension $F_{i}$, the strand stiffness $K$, and its unloaded length $L_{0}$. That is,

$$
d=\sqrt{L_{0}^{2}\left(1+\frac{F_{i}}{L_{0} K}\right)^{2}-\theta^{2} r^{2}}
$$

If $n$ strands are included in a string, the projected forces along vertical and radial directions can be derived, which are

$$
\begin{aligned}
& F_{i}=\frac{F_{z}}{n} \cdot \cos (\alpha), \\
& F_{\tau}=n \cdot F_{i} \sin (\alpha) .
\end{aligned}
$$

Thus, the generated torque $\tau_{L}$ becomes

$$
\tau_{L}=r \cdot F_{z} \cdot \tan (\alpha)=\frac{F_{z} \cdot \theta \cdot r^{2}}{d} .
$$

However, the total stroke of the linear movement of the string is restricted by the number of allowable revolutions of the string. Based on this geometric relationship, there exists a maximum angle (or revolutions) $\alpha_{\max }$ when all strands are wrapped tightly on each other. After this point, strands begin

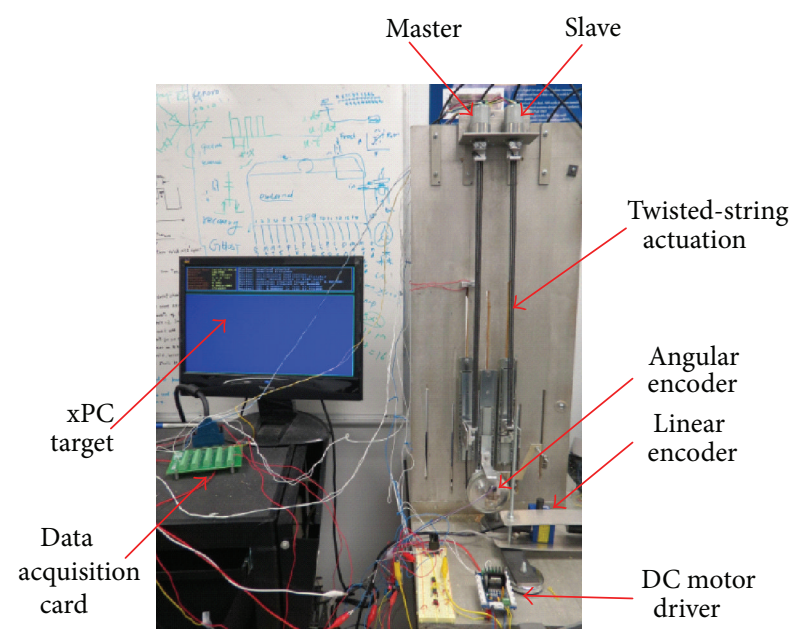

FIgURE 4: Configuration of experimental setup of a dual twistedstring actuation system.

entangling around themselves. Assuming there is no load $\left(F_{z}=0\right)$ applied to the transmission and combining (3) with the geometry of the strands at maximum $\theta$ angle, $\theta_{\max }$ and $d_{\text {min }}$ are

$$
\begin{aligned}
& \theta_{\max }=\frac{L_{0}}{\sqrt{r^{2}+\left(r_{s} \cdot n / \pi\right)}}, \\
& d_{\min }=\frac{L_{0}}{\sqrt{1+\left(r \cdot \pi / r_{s} \cdot n\right)^{2}}} .
\end{aligned}
$$

2.2. Physical Setup of Experimental Platform. In this study, a joint of a robotic system was adopted to test the tracking and synchronization of a dual twisted-string actuation system. The configuration of the actuation is shown in Figure 4. Two strings, each with 7 strands (1 neutral and 6 effective), were used to extend and contract the attached strings to drive the rotational movement of the joint. The diameter of each strand of adopted tactical cables is $1.2 \mathrm{~mm}$, and the original length of a single strand is $400 \mathrm{~mm}$. The extensional or contractile string was connected to two holders, as shown in Figure 5, at both ends with one end driven by a gear motor and one end connected to a belt that rotates the joint. The resolution of the encoders attached to the driving motors is 0.05 degrees, and the resolution of linear encoders used to measure the linear movements of the twisted strings is $0.04 \mathrm{~mm}$.

However, the rotational movements of the driving DC motors do not yield a proportional linear extension/contraction of the twisted strings. Instead, the linear extension/contraction is a position based profile of the angular movements of the driving motor. Though the nonlinear relationship between the DC motor and twisted sting can be obtained based on the model derived in the previous section, the cycle of a complete extension-contraction motion of the physical mechanism includes a hysteresis process, so there is not a single-valued function relating the motor revolutions to the length of the twisted string. To precisely control the 


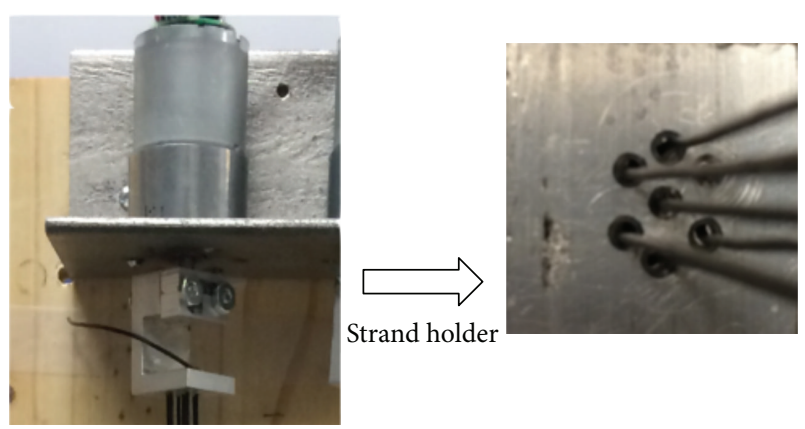

FIgURE 5: Alignment of an untwisted 7-strand string.

trajectory tracking and synchronization, it is required to verify the deviation between the theoretical estimation and actual result of the twisted strings. In the experiment, a $35 \mathrm{~N}$ load was applied to one of the actuation devices. To acquire the movement data, a desktop PC with a Pentium IV 2.4 GHz CPU was used for data acquisition. Two data acquisition I/O cards (PCI-6221 and PCI-6601) manufactured by National Instruments were used to send out command signals to the motor driver and to acquire signals generated by the optical encoders of the motor and the pulley. The data acquisition programs and control algorithm were developed using MATLAB Simulink with xPC Toolbox.

The original length of both 7-strand strings is $400 \mathrm{~mm}$. In the test, the string was first pretwisted to $300 \mathrm{~mm}$. It was then extended and contracted five times for a $60 \mathrm{~mm}$ stroke. Figure 6 demonstrates the comparison between experimental results and theoretical estimates for both contraction and extension. It shows a consistent repeatability of length variation. It is clear that a discrepancy exists between the theoretical estimates and the experimental results. The differences between the experimental outcomes and theoretical estimates can be due to the elastic behavior caused by the additional force created by the attached load. For instance, as the strands become tighter, the corresponding normal force yields larger friction, which can result in greater deviation between theoretical estimation and experimental result as the number of revolutions becomes higher. It is also clear that the relationship between motor revolutions and length variation of the string is not linear. A complete extension/contraction stroke of the string actuation is a hysteresis process. To reduce the hysteresis, it is required to pretwist the strings to tension them slightly instead of operating the strings from a slack situation. Thus, to make a smooth movement of the joint, a trajectory planning mechanism for both driving motors is necessary.

Other than nonlinear displacement, two other concerns need to be considered while using twisted-string actuation. One of them is the increased static friction. As the number of revolutions of the string increases, the normal force between contact surfaces increases as well. This also means that the driving DC motor needs to provide more torque to overcome the greater static friction due to the tightened cables. The other concern is that the string might start to kink if the string is twisted more than its maximum allowable revolutions. The kinking phenomenon is a nonlinear behavior that cannot be estimated with the model proposed in the previous section. From the theoretical model and the experimental results, the best operational range for a $60 \mathrm{~mm}$ stroke is suggested to be between 15 and 35 revolutions for the adopted tactical cable. Thus, to provide accurate trajectory tracking, path planning is necessary.

2.3. Model of Actuating DC Motor. The adopted driving devices that are used to drive the twisted strings are two typical DC motors. The transfer function from voltage command to the linear displacement of the twisted-string actuator can be represented by the following transfer function:

$$
G(s)=\frac{K_{0} \rho}{s(1+\tau s)},
$$

where

$$
\begin{aligned}
K_{0} & =\frac{K_{t}}{B R+K_{t} K_{b}}, \\
\tau & =\frac{J R}{B R+K_{t} K_{b}} .
\end{aligned}
$$

In this case, $J$ is the effective rotational inertia, $B$ is the effective viscous friction coefficient, $R$ is the resistance of motor armature, $K_{t}$ is the constant of motor torque constant, $K_{b}$ is motor back-EMF constant, and $\rho$ is the transmission ratio between the revolutions of DC motor, or $\alpha$, and displacement of the attached twisted string by performing a curve fitting within the selected operational range. In addition, the transmission ratio $\rho$ is not a constant. The ration is a position based function $\rho(\theta)$ of the revolutions of the driving motor. Based on (3) and (7), $\rho(\theta)$ can be further derived.

\section{Synchronization of a Dual Twisted-String Actuator and Cross-Coupled Dynamics}

As shown in Figure 4, the extending/contracting movement requires two twisted-string actuators to drive a single joint to rotate in two directions. However, since the two actuators are physically connected with each other, motion in one direction is affected by the motion actuated by the other string. In other words, the two actuators are cross-coupled by the physical connection. Thus, in order to precisely control the rotational movement of the joint and to synchronize the motions of both strings, cross-coupled dynamics becomes an important issue of controller synthesis. In particular, the relationship between motor turns $\alpha$ and $\Delta d$ of the two strings cannot be the same while one is extending and the other one is contracting due to the hysteresis and to the nonlinear relationship between $\alpha$ and $d$. Thus, as one motor starts to twist, the other one must untwist at a different rate. Poor synchronization can yield vibration of the joint. With this consideration, it is necessary to carefully plan the rotational movements of both motors according to the desired angular position of the joint. Lack of proper synchronization control between twisted-string actuators can lead to the whole system being unstable. 

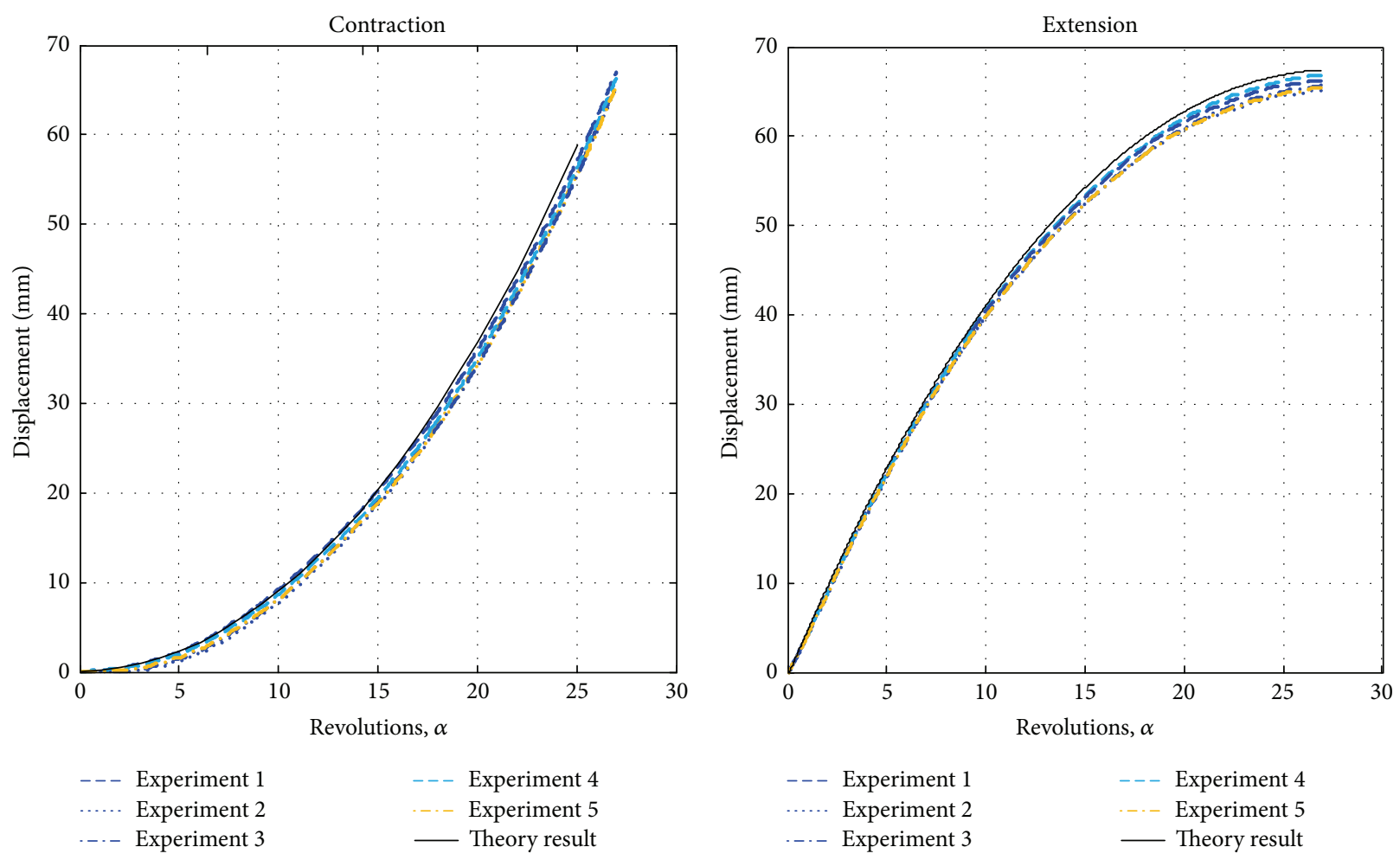

FIgURE 6: Comparison of theoretical transmission length and experimental measurement for $400 \mathrm{~mm}$ length.

To generate adequate trajectories for individual actuators, a path planning mechanism is required. The mechanism is in charge of converting the desired angular movement $\phi$ of a joint into the linear movements of the two strings. Then the required linear movement is converted into angular movements of individual driving motors, $\theta_{d 1}$ and $\theta_{d 2}$. The cross-coupled dynamics considered in this dual-axial motion system includes both tracking and synchronization. The feedback of tracking information includes the angular positions of both motors and the linear advance/retraction of both strings. To maintain the accuracy of rotational movement of a joint, the lengths of linear advance/retraction on both sides need to be synchronized. The error dynamics of individual string can then be defined as

$$
e_{i}=\theta_{i}-\theta_{d i}
$$

The synchronization error is determined by the deviation of actual lengths of the two strings, which can be written as

$$
e_{\text {syn }}=\left(\theta_{1}-\theta_{d 1}\right)-\left(\theta_{2}-\theta_{d 2}\right)=e_{1}-e_{2} .
$$

Thus, with (3) and (7), the disturbance force caused by crosscoupled dynamics can be obtained.

\section{Controller Synthesis with the Consideration of Cross-Coupling Dynamics}

To compensate for the tracking performance of the joint, the desired trajectory is converted to linear displacements of both actuators. The linear displacements of individual actuators are then converted to the corresponding revolutions of the driving motors.

In this section, three types of control structures will be investigated to reduce the effect of cross-coupled dynamics. They are (1) regular PID controller, (2) PID controller with the feedback of cross-coupling dynamics (PID-FC), and (3) LQR controller with the feedback of cross-coupling dynamics (LQR-FC). The goal of the controller is to compensate for both tracking and synchronizing performances of two twisted-string actuators. To achieve these objectives it is necessary to transfer the angular movement of the joint to linear movement of the string.

4.1. PID Controller. The controllers used in this section are two PID controllers designed using pole-placement method that compensates individual actuators independently. The controller synthesis is based on the model of the driving motors and the approximation of the twisted-string actuating mechanisms. Since the actuation systems of both sides adopt the same type of driving motors and tactical cable, the transfer functions used to design the controllers are the same. The trajectories of the individual motors are dispatched by a trajectory planning function depending on the desired angular position of the joint. Figure 7 illustrates the block diagram of the two twisted-string actuators. With the simulation results, the designed controller can provide adequate tracking performance for a single actuator if the desired poles are carefully selected. With this design, the two actuators are assumed to be two independent SISO systems that do not interfere with each other. The two actuators are designed 


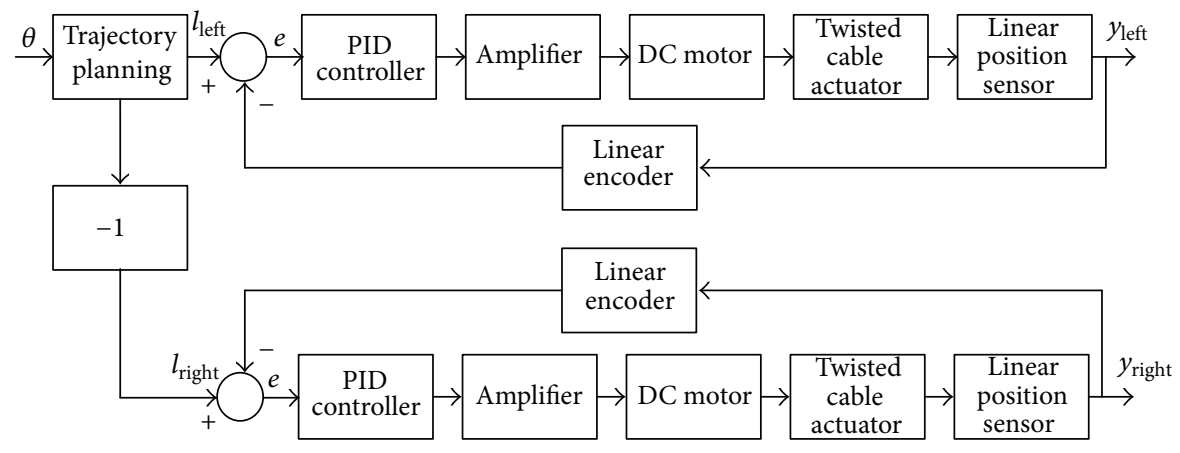

FIgURE 7: Block diagram of control scheme with regular PID controllers.

to track the opposite trajectory input, where a trajectory planning function is introduced to transfer a predefined angular movement to a trajectory of linear displacement. Instead of tracking the angular position of the driving motors, two linear encoders measuring the linear displacement of the two strings were adopted. In the experiments, the joint driven by the twisted-string actuating system is designed to track both sinusoidal and trapezoid signals which are converted to the linear displacement of two actuators.

To evaluate the performance of the controlled outcome, two types of motions were used: sinusoidal and trapezoidal movements. For the sinusoidal motion, the amplitude of the periodic displacement is $60 \mathrm{~mm}$ and the duration of each cycle is $4 \mathrm{sec}$. For the trapezoidal motion, each string extends/contracts $40 \mathrm{~mm}$ within $2 \mathrm{sec}$ from rest. It then stops for $2 \mathrm{sec}$ and then returns to its starting position within another $2 \mathrm{sec}$. Figures 8 and 9 illustrate the experimental results of the sinusoidal and trapezoidal motion tests, including linear displacements and synchronization errors. From the experiment results, it is clear that such an approach yields a poor performance in both tracking and synchronization without considering the cross-coupling dynamics between the two actuators. The maximum synchronization error can be as high as $20 \mathrm{~mm}$ for sinusoidal motion and $6 \mathrm{~mm}$ for trapezoidal motion. The huge error of both tracking and synchronization can be caused by disturbances created by the string actuator in the opposite direction. Such disturbances can also generate unevenly distributed loads on the twisted-string actuators at both sides. Therefore, the required amounts of contraction/extension for the two sides are often not the same, which can change the required revolution of the driving DC motors.

4.2. PID Controller with Feedback of Cross-Coupling Dynamics. As the motor on one side starts to twist one string, the string on the other side must untwist. Unsynchronized motion can make the hysteresis phenomenon of linear displacement of the string more severe so that both tracking and synchronization performances are degraded. In this case, regular PID controller cannot compensate for trajectory tracking precisely due to the disturbance and unsynchronized motion. In order to improve both tracking and synchronization performances, cross-coupling dynamics in the multiaxial system needs to be added to individual feedback loops. With

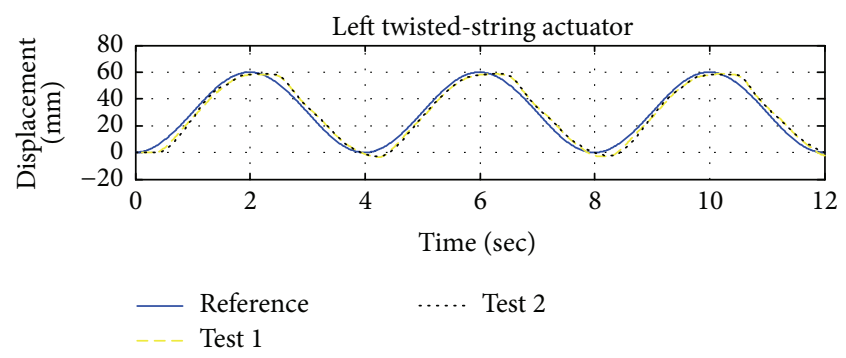

(a)

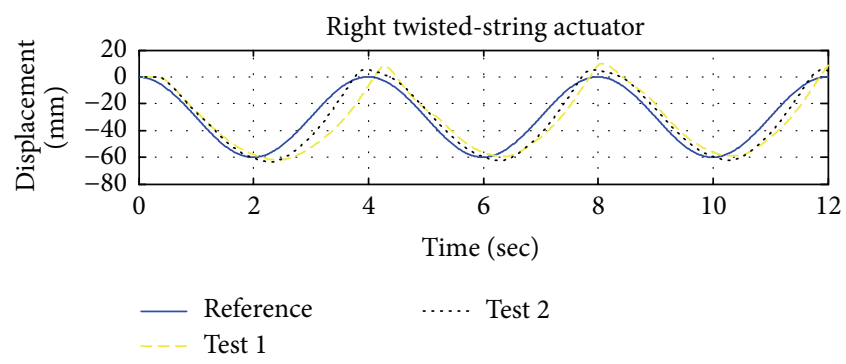

(b)

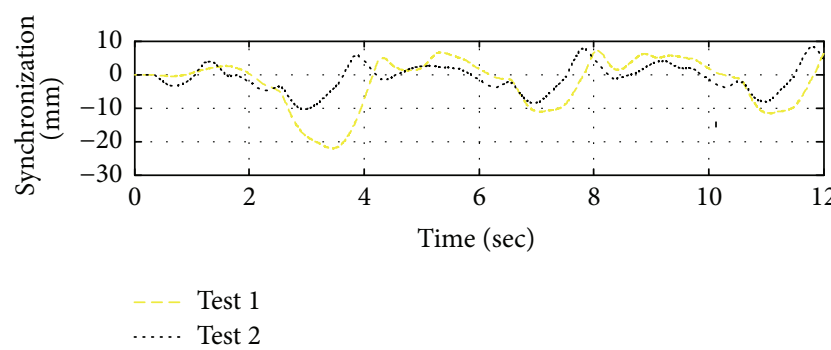

(c)

FIGURE 8: Experimental outcome of PID controller (sinusoidal motion): (a) comparison between reference trajectory and actual trajectories of left actuator; (b) comparison between reference trajectory and actual trajectories of right actuator; (c) synchronization errors between left and right actuators.

the consideration of cross-coupling dynamics, the control structure can be modified as shown in Figure 10. The crosscoupled dynamics adopted in this study is the deviation of the linear positions of both actuators with a gain. The control goal of such a control strategy is to make both tracking 


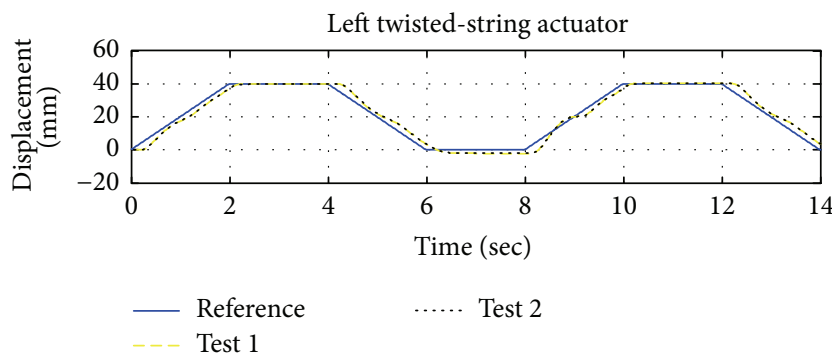

(a)

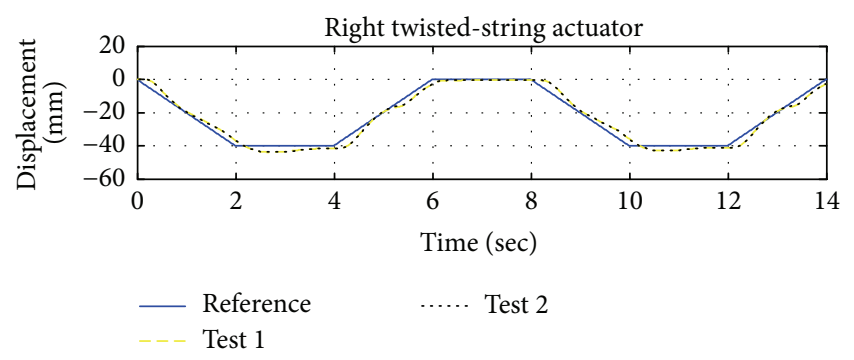

(b)

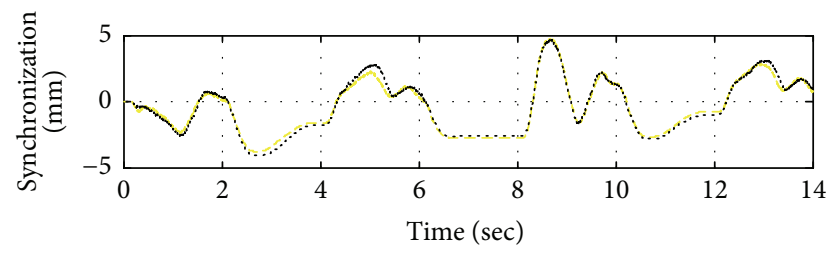

- Test 1

….. Test 2

(c)

Figure 9: Experimental outcome of PID controller (trapezoidal motion): (a) comparison between reference trajectory and actual trajectories of left actuator; (b) comparison between reference trajectory and actual trajectories of right actuator; (c) synchronization errors between left and right twisted actuators.

and synchronization errors converge to zero. In this case, synchronization error between two actuators is introduced as input disturbance in the feedback loop with a crosscoupling gain. Then the cross-coupling gain can be adjusted according to its importance. High gain compensation of synchronization error due to cross-coupled relationship can compensate for the synchronization performance. However, the trade-off is that the performance of tracking controller of individual axes can be less effective since more control effort is focused on synchronization. Meanwhile, compensation of synchronization error can also result in instability if the gain is too high.

Figures 11 and 12 illustrate the controlled results of PIDFC. It is clear that the maximal synchronization error can be dramatically reduced to only less than $3 \mathrm{~mm}$ for the desired sinusoidal motion. It also improves tracking error to less than $1 \mathrm{~mm}$ for trapezoidal trajectory as well. However, the gain of cross-coupling control system design is generally a trail-anderror process in which the design parameters are determined by comparing the performance among different parameters.
4.3. LQR Controller with Feedback of Cross-Coupling Dynamics. To compensate for trajectory tracking of multiaxial systems, linear quadratic optimal control law has been found to be an effective mathematic algorithm that minimizes a cost function with specified design goals. Cheng et al. [16] have proposed a generalized framework in which multiaxis motion systems can be represented as multiple subsystems with dynamics coupling among them. To apply LQR method to such a cross-coupled dual-axis system, the state-space presentation of two actuators is given by

$$
\begin{aligned}
& \dot{\mathbf{d}}(t)=\mathbf{A} \cdot \mathbf{d}(t)+\mathbf{B} \cdot \mathbf{u}(t), \\
& \mathbf{y}(t)=\mathbf{C} \cdot \mathbf{d}(t),
\end{aligned}
$$

where $t$ is time, $\mathbf{d}(t)$ and $\mathbf{u}(t)$ are the vectors of state variables and control inputs, and $\mathbf{A}$ and $\mathbf{B}$ are system matrices in the following form:

$$
\begin{aligned}
& {\left[\begin{array}{l}
\dot{d}_{1}(t) \\
\ddot{d}_{1}(t) \\
\dot{d}_{2}(t) \\
\ddot{d}_{2}(t)
\end{array}\right]=\left[\begin{array}{cccc}
0 & 1 & 0 & 0 \\
0 & -\frac{1}{\tau_{1}} & 0 & 0 \\
0 & 0 & 0 & 1 \\
0 & 0 & 0 & -\frac{1}{\tau_{2}}
\end{array}\right]\left[\begin{array}{l}
d_{1}(t) \\
\dot{d}_{1}(t) \\
d_{2}(t) \\
\dot{d}_{2}(t)
\end{array}\right]} \\
& +\left[\begin{array}{cc}
0 & 0 \\
\frac{K_{01}}{\tau_{1}} & 0 \\
0 & 0 \\
0 & \frac{K_{02}}{\tau_{2}}
\end{array}\right]\left[\begin{array}{l}
u_{1}(t) \\
u_{2}(t)
\end{array}\right] \\
& \mathbf{C}=\left[\begin{array}{llll}
1 & 0 & 0 & 0 \\
0 & 0 & 1 & 0
\end{array}\right] \text {. }
\end{aligned}
$$

In this study, the actuators are two independent motors. Thus, the coefficients of the matrices $a_{i j}$ and $b_{i j}(i \neq j)$ are all null matrices. $\tau_{1}, \tau_{2}, K_{01}$, and $K_{02}$ are the physical parameters of individual twisted-string actuators. Since the goal is to regulate the linear displacement, $d_{1}$ and $d_{2}$, as well as the difference between these two displacements, a linear quadratic (LQ) optimal control method is applied to reduce the synchronization error. In order to achieve accurate tracking and synchronization performance, the performance criteria, in which both tracking and synchronization errors need to be considered, can be expressed as the following equation:

$$
\begin{aligned}
& J_{p} \\
& \quad=\int_{t_{0}}^{t_{f}}\left[\sum_{i=1}^{2} q_{i} \cdot d_{i}(t)^{2}+\sum_{j=1}^{2} r_{j} \cdot u_{j}^{2}+\kappa\left(d_{1}-d_{2}\right)^{2}\right] d t,
\end{aligned}
$$

where $q_{i}, r_{i}$, and $\kappa$ are the parameters that need to be determined. To consider the feedback cross-coupling dynamics, the deviations of the linear displacements of both actuators are used. The synchronization error can then be transformed 


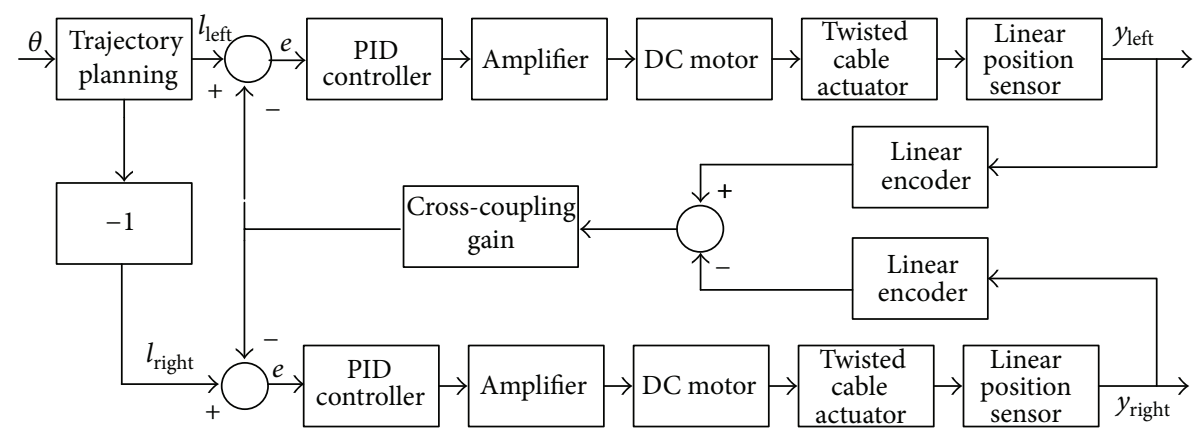

FIGURE 10: Block diagram of PID-FC controllers.

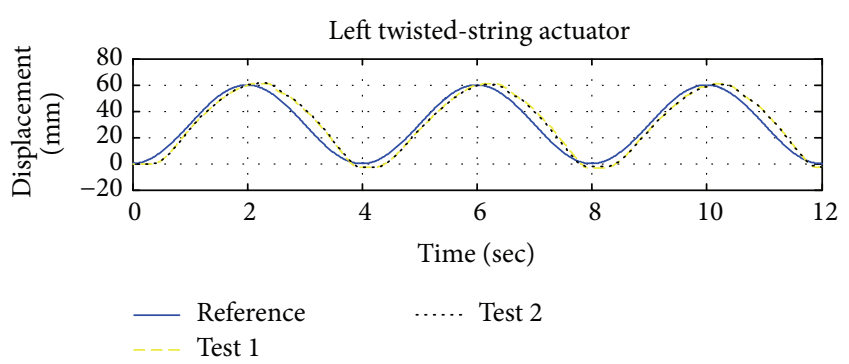

(a)

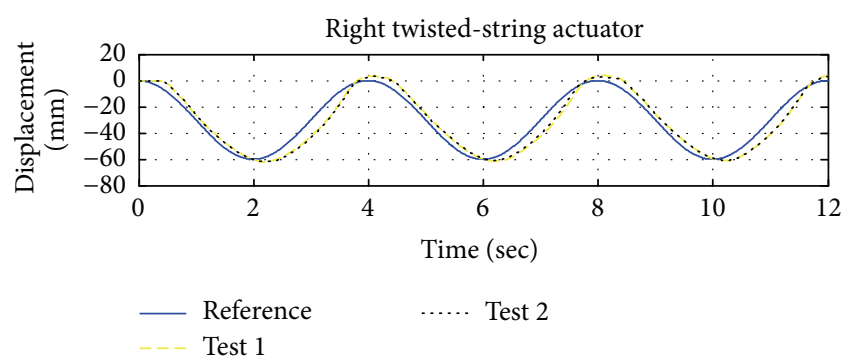

(b)

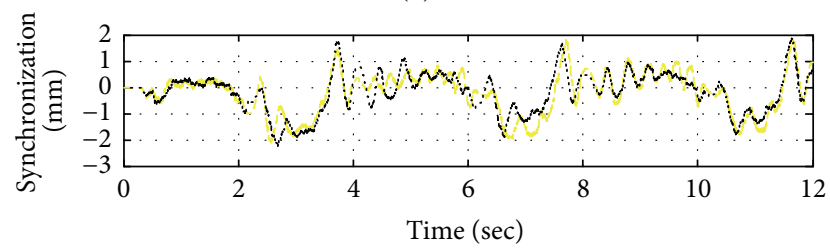

Test 1

..... Test 2

(c)

FIGURE 11: Experimental outcome of PID-FC controller (sinusoidal motion): (a) comparison between reference trajectory and actual trajectories of left actuator; (b) comparison between reference trajectory and actual trajectories of right actuator; (c) synchronization errors between left and right twisted actuators.

to the representation of state variables by selecting the specified $\mathbf{Q}$ matrix. The $\mathbf{Q}$ matrix can be defined as

Q

$$
=\left[\begin{array}{cccc}
1 & 0 & \frac{\sqrt{2}}{2} & \frac{\sqrt{2}}{2} \\
0 & 0 & 0 & 0 \\
0 & 1 & -\frac{\sqrt{2}}{2} & -\frac{\sqrt{2}}{2} \\
0 & 0 & 0 & 0
\end{array}\right]\left[\begin{array}{cccc}
q_{1} & 0 & 0 & 0 \\
0 & q_{2} & 0 & 0 \\
0 & 0 & \rho & 0 \\
0 & 0 & 0 & \rho
\end{array}\right]\left[\begin{array}{cccc}
1 & 0 & 0 & 0 \\
0 & 0 & 1 & 0 \\
\frac{\sqrt{2}}{2} & 0 & -\frac{\sqrt{2}}{2} & 0 \\
\frac{\sqrt{2}}{2} & 0 & -\frac{\sqrt{2}}{2} & 0
\end{array}\right],
$$

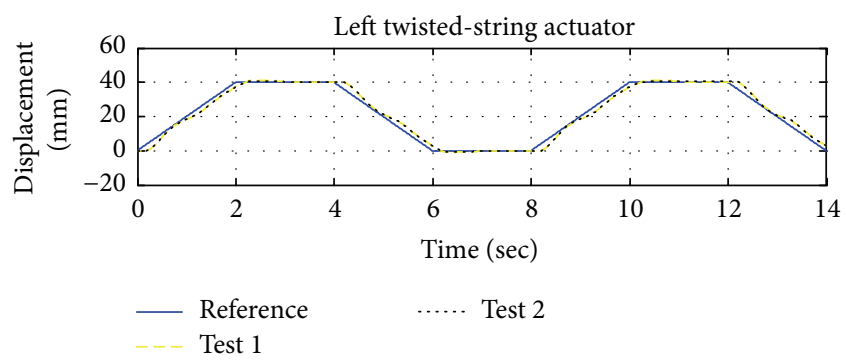

(a)

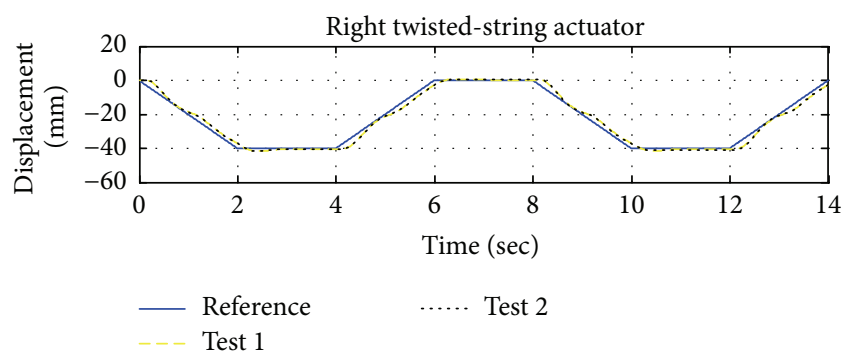

(b)

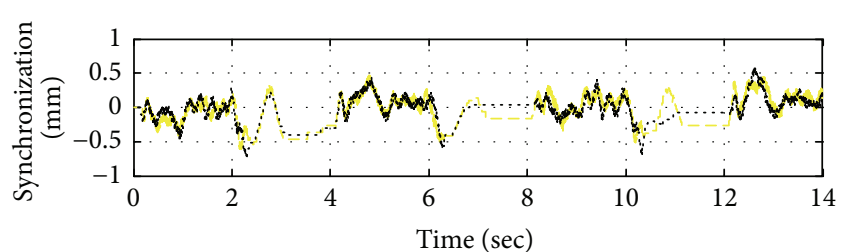

Test 1

….. Test 2

(c)

FIGURE 12: Experimental outcome of PID-FC controller (trapezoidal motion): (a) comparison between reference trajectory and actual trajectories of left actuator; (b) comparison between reference trajectory and actual trajectories of right actuator; (c) synchronization errors between left and right twisted actuators.

and the $\mathbf{R}$ matrix is

$$
\mathbf{R}=\left[\begin{array}{ll}
r_{1} & 0 \\
0 & r_{2}
\end{array}\right] .
$$

The selection of weight matrices $\mathbf{Q}$ and $\mathbf{R}$ in linear quadratic optimal control is very important and they have direct effect on controller's performance. In general, $\mathbf{Q}$ and $\mathbf{R}$ matrices 
are determined based on experience. Therefore, the controller might not be truly optimal or possibly be unstable. To ensure the best performance, genetic algorithm (GA) based on Darwin's theories was used to search for the coefficients of $\mathbf{Q}$ and $\mathbf{R}$ matrices for global optimal. In this study, the $\mathbf{Q}$ and $\mathbf{R}$ matrices are

$$
\begin{aligned}
& \mathbf{Q}=\left[\begin{array}{cccc}
4975 & 0 & -2189 & 0 \\
0 & 0 & 0 & 0 \\
-2189 & 0 & 4975 & 0 \\
0 & 0 & 0 & 0
\end{array}\right], \\
& \mathbf{R}=\left[\begin{array}{cc}
0.4978 & 0 \\
0 & 0.4978
\end{array}\right]
\end{aligned}
$$

Once the $\mathbf{Q}$ and $\mathbf{R}$ matrices have been determined, the optimal control law is given by

$$
\mathbf{K}=\left[\begin{array}{cccc}
97.38 & 4.23 & -22.57 & -0.53 \\
-22.57 & -0.53 & 97.38 & 4.23
\end{array}\right] \text {. }
$$

The control law is derived to drive the output, or the state, to 0 . However, in this case, the objective of the controller is to drive the joint to track a reference input. For $r(t)$ is the reference input, the goal is to make $y(t) \rightarrow r(t)$ as $t \rightarrow \infty$. To achieve this objective, a feedforward controller is required. We first design a steady state vector $\mathbf{d}_{\mathrm{ss}}=\mathbf{N}_{\mathbf{x}} \cdot r$ for constant reference input $r$, in which $\mathbf{N}_{\mathbf{x}}$ converts the reference inputs $r$ into the desired values of the states. The new control law can be defined as

$$
\mathbf{u}=-\mathbf{K}\left(\mathbf{d}-\mathbf{d}_{\mathrm{ss}}\right)+\mathbf{u}_{\mathrm{ss}},
$$

where $\mathbf{u}_{\mathrm{ss}}=\mathbf{N}_{\mathbf{u}} \cdot r$ is the steady state control input to maintain $\mathbf{d}$ at $\mathbf{d}_{\mathrm{ss}}$. To find $\mathbf{N}_{\mathbf{x}}$ and $\mathbf{N}_{\mathbf{u}}$, we define the desired steady state relationships:

$$
\begin{aligned}
\dot{\mathbf{d}}_{\mathrm{ss}}(t) & =\mathbf{A} \cdot \mathbf{d}_{\mathrm{ss}}(t)+\mathbf{B} \cdot \mathbf{u}_{\mathrm{ss}}(t)=\left(\mathbf{A} \cdot \mathbf{N}_{\mathbf{x}}+\mathbf{B} \cdot \mathbf{N}_{\mathbf{u}}\right) \mathbf{r} \\
& =0 \\
\mathbf{y}(t) & =\mathbf{C} \cdot \mathbf{d}_{\mathrm{ss}}(t)=\left(\mathbf{C} \cdot \mathbf{N}_{\mathbf{x}}+0\right) \mathbf{r}=\mathbf{r} .
\end{aligned}
$$

To make this work for all $r$, we need to solve

$$
\left[\begin{array}{ll}
A & B \\
C & 0
\end{array}\right]\left[\begin{array}{l}
\mathbf{N}_{\mathbf{x}} \\
\mathbf{N}_{\mathbf{u}}
\end{array}\right]=\left[\begin{array}{l}
\mathbf{0} \\
\mathbf{I}
\end{array}\right]
$$

Substituting the values of $\mathbf{A}, \mathbf{B}$, and $\mathbf{C}$ gives

$$
\begin{aligned}
& \mathbf{N}_{\mathbf{u}}=\mathbf{0}, \quad \mathbf{N}_{\mathbf{u}} \in R^{2 \times 2}, \\
& \mathbf{N}_{\mathbf{x}}=\left[\begin{array}{llll}
1 & 0 & 0 & 0 \\
0 & 0 & 1 & 0
\end{array}\right]^{T}, \quad \mathbf{N}_{\mathbf{x}} \in R^{4 \times 2} .
\end{aligned}
$$

Once $\mathbf{N}_{\mathbf{x}}$ and $\mathbf{N}_{\mathbf{u}}$ were found, we can rewrite the control law (19) to be

$$
\mathbf{u}=\mathbf{g} \cdot \mathbf{r}-\mathbf{K} \cdot \mathbf{d},
$$

TABLE 1: Comparison of performance of controllers of sinusoidal motion.

\begin{tabular}{lcc}
\hline & \multicolumn{2}{c}{ RMSE (mm) } \\
& Sync. error & Tracking error \\
\hline \multirow{2}{*}{ PID without cross-coupling } & \multirow{2}{*}{3.88} & $4.03(\mathrm{~L})$ \\
& & $6.92(\mathrm{R})$ \\
\hline \multirow{2}{*}{ PID-FC } & \multirow{2}{*}{0.62} & $5.03(\mathrm{~L})$ \\
& & $5.32(\mathrm{R})$ \\
\hline \multirow{2}{*}{ LQR-FC } & \multirow{2}{*}{0.63} & $1.66(\mathrm{~L})$ \\
& & $1.65(\mathrm{R})$ \\
\hline
\end{tabular}

where $\mathbf{g}$ is the feedforward gain. That is,

$$
g=N_{u}+K_{x}=\left[\begin{array}{ll}
K & I
\end{array}\right]\left[\begin{array}{ll}
A & B \\
C & 0
\end{array}\right]^{-1}\left[\begin{array}{l}
0 \\
I
\end{array}\right] .
$$

However, this feedforward controller only accounts for the constant reference input because $\mathbf{N}_{\mathbf{x}}$ and $\mathbf{N}_{\mathbf{u}}$ rely on modeling of the plant which might not be accurate and LQR is a kind of optimal controllers which is not focusing on disturbance rejection. To track the time varying reference input and reject disturbances, integral control can be added to the system. This can be achieved by augmenting the system by the integrator state $\mathbf{d}_{\mathbf{I}}$ :

$$
\left[\begin{array}{c}
\dot{d}_{I}(t) \\
\dot{d}(t)
\end{array}\right]=\left[\begin{array}{cc}
0 & -C \\
0 & A
\end{array}\right]\left[\begin{array}{l}
d_{I}(t) \\
d(t)
\end{array}\right]+\left[\begin{array}{l}
0 \\
B
\end{array}\right] \mathbf{u}+\left[\begin{array}{l}
I \\
0
\end{array}\right] \mathbf{r}(t) .
$$

Since $\mathbf{N}_{\mathbf{u}}$ is equal to zero, the final control law is

$$
\mathbf{u}=-\left[\begin{array}{ll}
\mathbf{K}_{\mathbf{I}} & \mathbf{K}
\end{array}\right]\left[\begin{array}{l}
\mathbf{d}_{\mathbf{I}} \\
\mathbf{d}
\end{array}\right]+\mathbf{K N}_{\mathbf{x}} \mathbf{r}(\mathbf{t}),
$$

where $\mathbf{K}_{\mathbf{I}}$ is integral gain. With this technique, the disturbance can be rejected effectively as well [17]. Figures 13 and 14 demonstrate the experimental results of tracking performance for both sinusoidal and trapezoidal motions. It is clear that the LQR-FC controller can improve both the transient and steady state tracking performances over those of the PID controllers. With the LQR-FC approach, the synchronization performance can be improved as well. It can be observed that the maximum synchronization error can be restricted to within $2 \mathrm{~mm}$, which is slightly better than the outcome of PID-FC controller. It also demonstrates better tracking performance than PID-FC controller.

4.4. Comparison of Controllers. Based on different types of controllers, the tracking performances of the dual twistedstring actuating systems are different as well. Among the three controllers, LQR-FC has the smallest synchronization and tracking errors. Table 1 lists the comparison of both tracking and synchronization errors (root mean-square and maximum errors) among all three controllers. It is clear that the PID controller is not able to provide satisfactory tracking performance while the other two types of controllers, PIDFC and LQR-FC, have significantly smaller errors. It is also 
TABLE 2: Time delay of the three controllers of sinusoidal motion.

\begin{tabular}{lc}
\hline & Delay (sec) \\
\hline \multirow{2}{*}{ PID without cross-coupling } & $0.37(\mathrm{~L})$ \\
& $0.37(\mathrm{R})$ \\
\hline \multirow{2}{*}{ PID-FC } & $0.14(\mathrm{~L})$ \\
& $0.28(\mathrm{R})$ \\
\hline \multirow{2}{*}{ LQR-FC } & $0.08(\mathrm{~L})$ \\
& $0.02(\mathrm{R})$ \\
\hline
\end{tabular}

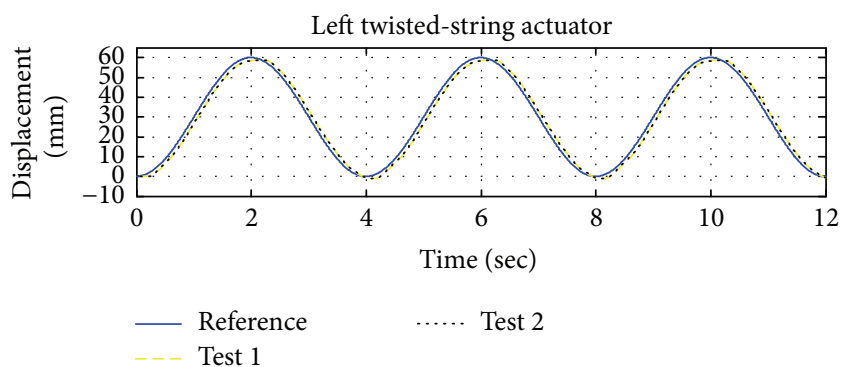

(a)
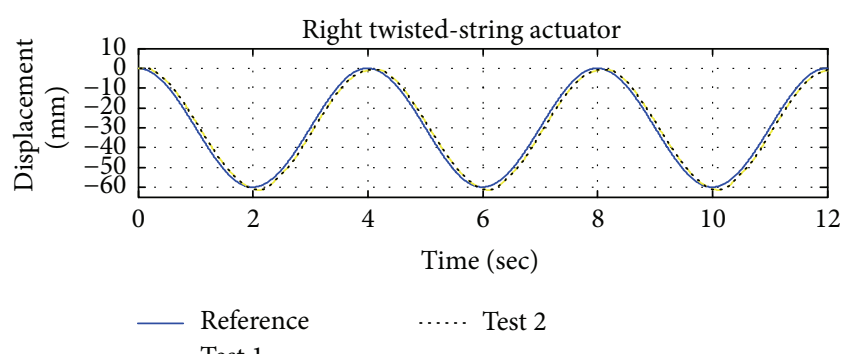

(b)

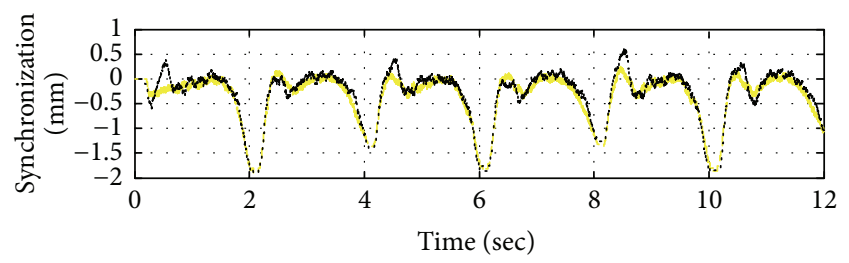

Test 1

….. Test 2

(c)

FIGURE 13: Experimental outcome of LQR-FC controller (sinusoidal motion): (a) comparison between reference trajectory and actual trajectories of left actuator; (b) comparison between reference trajectory and actual trajectories of right actuator; (c) synchronization errors between left and right actuators.

noticed that all three controlled systems have a slightly delayed response. Table 2 lists the time delays of three controllers. Longer time delay can yield greater tracking error. Among all the controllers, the LQR-FC has a significantly smaller time delay than the other two controllers. Similarly, the PID-FC uses the cross-coupling feedback to obtain a much smaller synchronization error than that of the plain PID controller. This also implies that PID-FC can have better

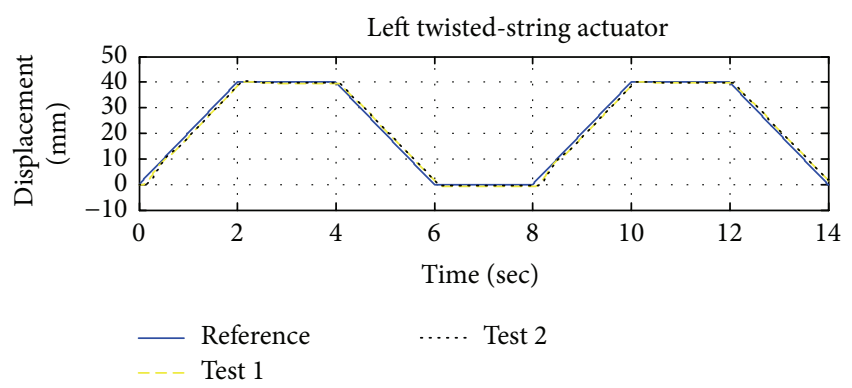

(a)

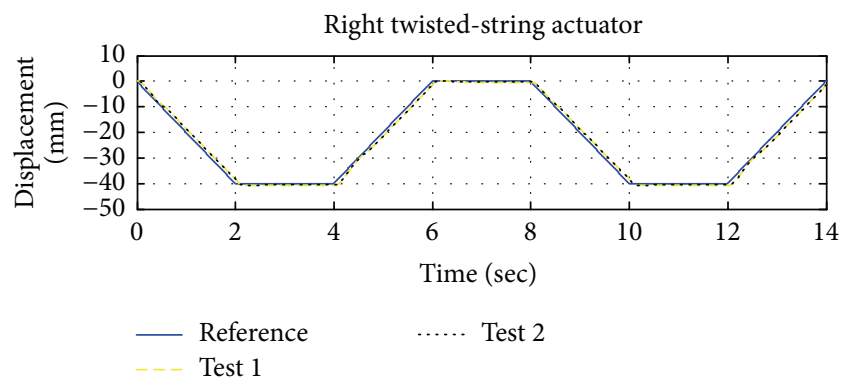

(b)

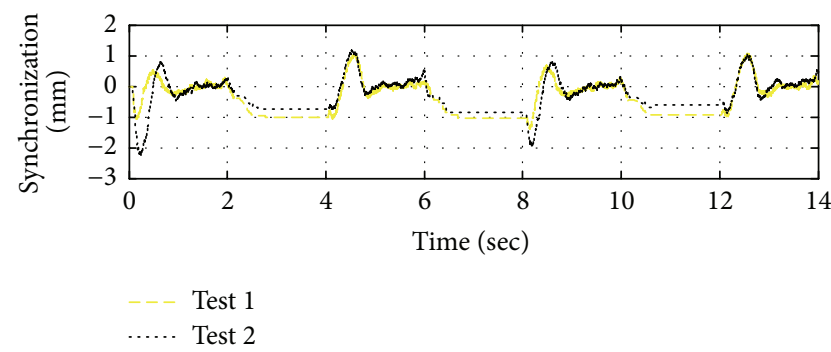

(c)

FIGURE 14: Experimental outcome of LQR-FC controller (trapezoidal motion): (a) comparison between reference trajectory and actual trajectories of left actuator; (b) comparison between reference trajectory and actual trajectories of right actuator; (c) synchronization errors between left and right actuators.

disturbance rejection if the disturbance is caused by the connected actuators.

To compare the performance between PID-FC and LQRFC controllers, Figure 15 illustrates tracking and synchronization errors of three full-cycle movements of these two controllers. The arrows indicate the directions of displacements of string actuation. Both controllers demonstrate good repeatability of tracking outcomes. Although the synchronization errors are not significantly different, the LQR-FC demonstrates a better tracking performance with its smaller root mean-square error (RMSE). This can be due to the greater delay of PID-FC caused by the structure of different controllers.

4.5. Discussion of Disturbance Rejection. From the previous sections, it is clear that the PID controller alone does not provide satisfactory tracking performance. To test disturbance rejection, only PID-FC and LQR-FC controllers were considered. In particular, disturbance rejection has been 

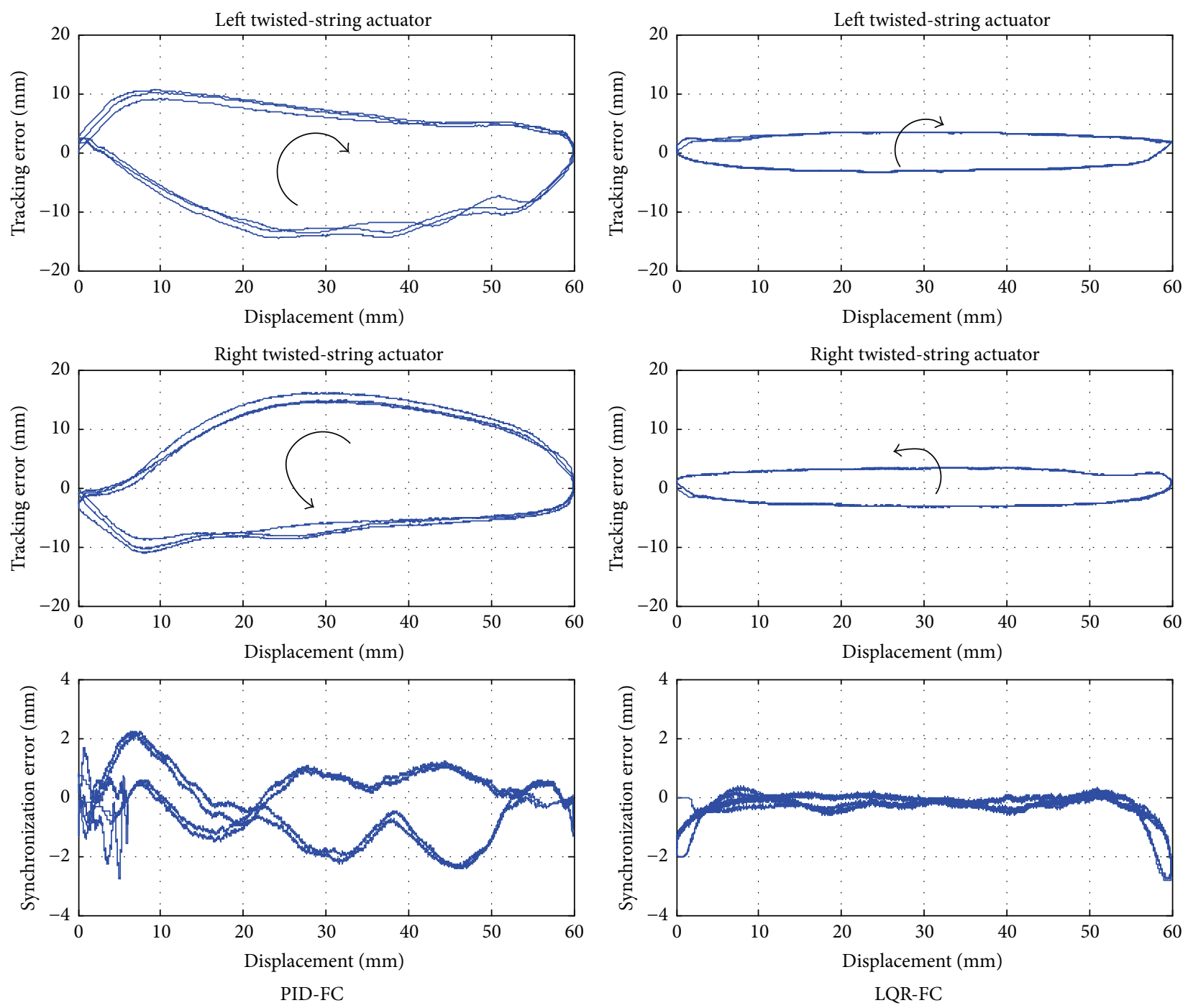

FIGURE 15: Comparison of tracking and synchronization errors of PID-FC and LQR-FC controllers.

considered and integrated while synthesizing the LQR-FC controller. In this section, two types of disturbance were applied to the dual twisted-string actuation joint. They are (1) a fixed $500 \mathrm{~g}$ loading and (2) a $500 \mathrm{~g}$ loading attached to the mechanism when the linear displacement of the string actuation is at a specific location. In this case, the displacement was at $40 \mathrm{~mm}$.

Figure 16 illustrates the experimental results of the controllers with a fixed $500 \mathrm{~g}$, or $4.91 \mathrm{~N}$, loading attached to the joint on the left actuator while moving, and a $500 \mathrm{~g}$ loading attached to the left actuator when the displacement of the string actuation is $40 \mathrm{~mm}$. From the experimental results, the tacking errors of the individual axes were affected slightly. There is no significant difference of tracking and synchronization performance between LQR-FC and PID-FC controllers. However, the PID-FC does have a longer time delay which yields greater tracking error. This error can be as large as $12 \mathrm{~mm}$ on one side. And the disturbance caused by the attached mass at a specific location does not generate a clear impact to the movement.
Figure 17 demonstrates the position based errors of both controllers for a complete cycle. In this figure, it is clear that LQR-FC can have a better tracking performance which also results in a slightly better synchronization performance due to its smaller time delay when a fixed loading or periodic loading is attached. Synchronization errors started to increase as a $500 \mathrm{~g}$ loading was attached at a specific displacement for both LQR-FC and PID-FC controllers. Such errors became smaller as the $500 \mathrm{~g}$ was removed from this specific location.

From the experimental results, the LQR-FC demonstrates smaller deviation from the desired trajectories for both types of disturbances. With the current setup, both tracking and synchronization performances can be guaranteed regardless of the load disturbances as tested here. Table 3 lists the performance of both controllers under two different scenarios.

\section{Conclusion}

To precisely compensate for the tracking performance of a single axial system, both PID and LQR algorithms can 


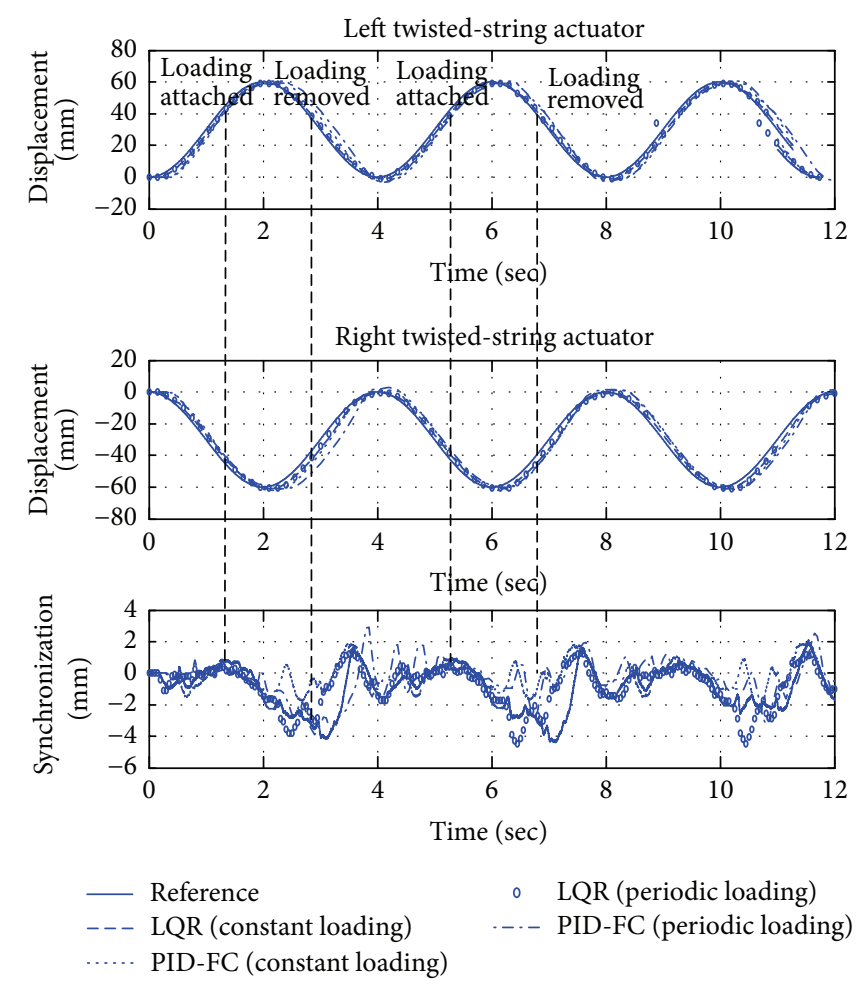

FIGURE 16: Tracking and synchronization performance of LQR-FC controller with $500 \mathrm{~g}$ fixed loading and periodic loading.
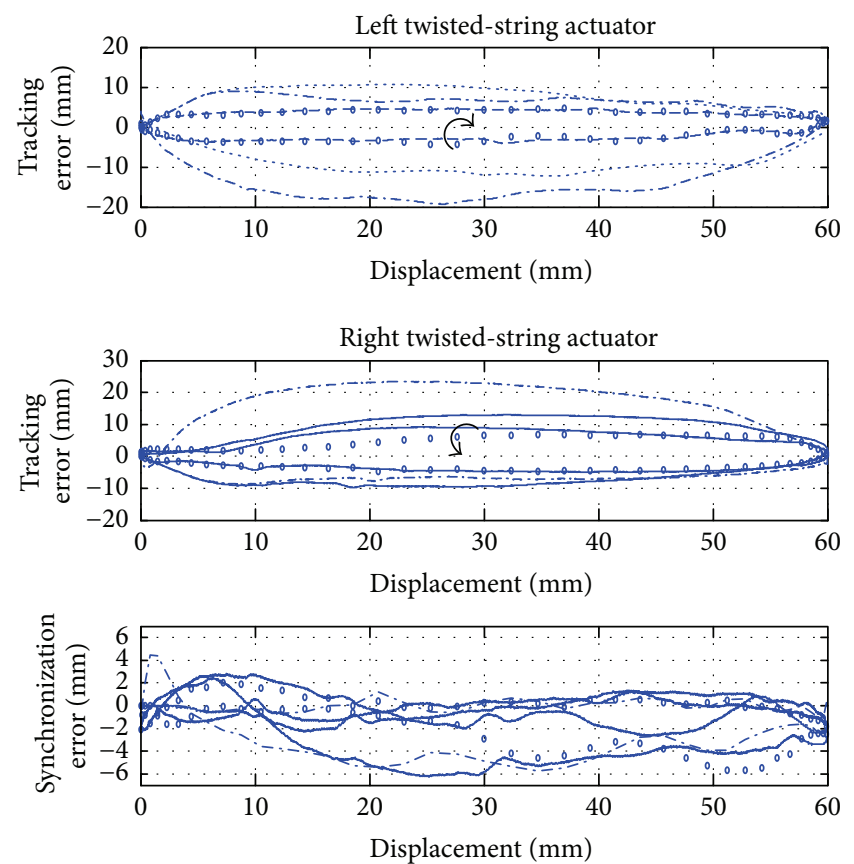

$\begin{array}{llll}\text { - } & \text { LQR (constant loading) } & \circ & \text { LQR (periodic loading) } \\ \ldots . . & \text { PID-FC (constant loading) } & -.-- & \text { PID-FC (periodic loading) }\end{array}$

FIGURE 17: Comparison of tracking and synchronization errors of PID-FC and LQR-FC controlled results with $500 \mathrm{~g}$ fixed loading and periodic loading.
TABLE 3: Comparison of the disturbance rejection between PID-FC and LQR-FC controllers with a $500 \mathrm{~g}$ loading.

\begin{tabular}{lcc}
\hline & $\begin{array}{c}\text { Maximum tracking } \\
\text { error }(\mathrm{mm})\end{array}$ & $\begin{array}{c}\text { Maximum } \\
\text { synchronization error } \\
(\mathrm{mm})\end{array}$ \\
\hline $\begin{array}{l}\text { PID-FC with a fixed } \\
500 \text { g loading }\end{array}$ & $\begin{array}{c}11.55(\mathrm{~L}) \\
12.56(\mathrm{R})\end{array}$ & 1.23 \\
\hline $\begin{array}{l}\text { PID-FC with a 500 g } \\
\text { loading attached at }\end{array}$ & $12.07(\mathrm{~L})$ & 2.79 \\
$40 \mathrm{~mm}$ & $13.01(\mathrm{R})$ & \\
\hline $\begin{array}{l}\text { LQR-FC with a fixed } \\
500 \text { g loading }\end{array}$ & $4.32(\mathrm{~L})$ & 3.87 \\
\hline $\begin{array}{l}\text { LQR-FC with a 500g } \\
\text { loading attached at }\end{array}$ & $4.01(\mathrm{R})$ & 6.12 \\
$40 \mathrm{~mm}$ & $9.08(\mathrm{R})$ & \\
\hline
\end{tabular}

generate satisfactory results. However, the controlled results can be fairly inaccurate if the cross-coupled relationships between individual actuators are not carefully compensated for a multiaxial system. This paper demonstrates two effective approaches to eliminate the deviation caused by such a relationship. With the consideration of cross-coupling dynamics, the proposed PID-FC and LQR-FC controllers can greatly reduce the synchronization errors between the two axes. If the delay is especially pernicious in the system, the PIDFC controller cannot satisfy the requirements for tracking performance of a twisted-string actuation system. Thus, the LQR-FC controller can provide a more adequate performance for both tracking and synchronization.

\section{Competing Interests}

The authors declare that they have no competing interests.

\section{References}

[1] T. Würtz, C. May, B. Holz, C. Natale, G. Palli, and C. Melchiorri, "The twisted string actuation system: modeling and control," in Proceedings of the IEEE/ASME International Conference on Advanced Intelligent Mechatronics (AIM '10), pp. 1215-1220, IEEE, Montreal, Canada, July 2010.

[2] G. Palli, C. Natale, C. May, C. Melchiorri, and T. Würtz, "Modeling and control of the twisted string actuation system," IEEE/ASME Transactions on Mechatronics, vol. 18, no. 2, pp. 664-673, 2013.

[3] G. Palli, M. Hosseini, L. Moriello, and C. Melchiorri, "Modeling and identification of a variable stiffness joint based on twisted string actuators," in Proceedings of the IEEE/RSJ International Conference on Intelligent Robots and Systems (IROS '15), pp. 1757-1762, IEEE, Hamburg, Germany, September-October 2015.

[4] G. Palli, L. Pan, M. Hosseini, L. Moriello, and C. Melchiorri, "Feedback linearization of variable stiffness joints based on twisted string actuators," in Proceedings of the IEEE International Conference on Robotics and Automation (ICRA '15), pp. 2742-2747, Seattle, Wash, USA, May 2015. 
[5] Y. J. Shin, H. J. Lee, K.-S. Kim, and S. Kim, "A Robot Finger design using a dual-mode twisting mechanism to achieve highspeed motion and large grasping force," IEEE Transactions on Robotics, vol. 28, no. 6, pp. 1398-1405, 2012.

[6] G. Palli, C. Melchiorri, G. Vassura et al., "Innovative technologies for the next generation of robotic hands," in Advanced Bimanual Manipulation, vol. 80 of Springer Tracts in Advanced Robotics (STAR), pp. 173-218, Springer, 2012.

[7] M. Tomizuka, J.-S. Hu, T.-C. Chiu, and T. Kamano, "Synchronization of two motion control axes under adaptive feedforward control," Journal of Dynamic Systems, Measurement and Control, vol. 114, no. 2, pp. 196-203, 1992.

[8] Y. Yokokohji and T. Yoshikawa, "Bilateral control of masterslave manipulators for ideal kinesthetic coupling-formulation and experiment," IEEE Transactions on Robotics and Automation, vol. 10, no. 5, pp. 605-620, 1994.

[9] N. Tsujiuchi, T. Koizumi, S. Nishino, H. Komatsubara, T. Kudawara, and M. Hirano, "Development of pneumatic robot hand and construction of master-slave system," Journal of System Design and Dynamics, vol. 2, no. 6, pp. 1306-1315, 2008.

[10] Y. Koren, "Cross-coupled biaxial computer control for manufacturing systems," Journal of Dynamic Systems, Measurement and Control, vol. 102, no. 4, pp. 265-272, 1980.

[11] G. T.-C. Chiu and M. Tomizuka, "Coordinated position control of multi-axis mechanical systems," Journal of Dynamic Systems, Measurement and Control, vol. 120, no. 3, pp. 389-393, 1998.

[12] S.-K. Jeong and S.-S. You, "Precise position synchronous control of multi-axis servo system," Mechatronics, vol. 18, no. 3, pp. 129140, 2008.

[13] M. H. Cheng, Y. J. Li, and E. G. Bakhoum, "Controller synthesis of tracking and synchronization for multiaxis motion system," IEEE Transactions on Control Systems Technology, vol. 22, no. 1, pp. 378-386, 2014.

[14] M. H. Cheng and E. G. Bakhoum, "Adaptive robust control of tracking and synchronization for multi-axis motion system," in Proceedings of the American Control Conference (ACC '11), pp. 1-6, July 2011.

[15] M. H.-M. Cheng, A. Mitra, and C.-Y. Chen, "Synchronization controller synthesis of multi-axis motion system," in Proceedings of the 4th International Conference on Innovative Computing, Information and Control (ICICIC '09), pp. 918-921, Kaohsiung, Taiwan, December 2009.

[16] M. H.-M. Cheng, C.-Y. Chen, and E. G. Bakhoum, "Synchronization controller synthesis of multi-axis motion system," International Journal of Innovative Computing, Information and Control, vol. 7, no. 7, pp. 4395-4410, 2011.

[17] T. Chen and B. Francis, Optimal Sampled-Data Control Systems, Springer, London, UK, 1995. 


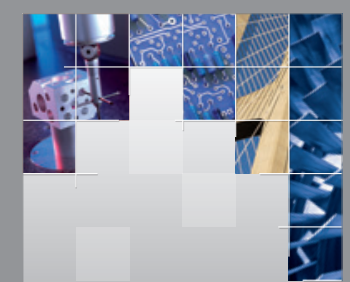

\section{Enfincering}
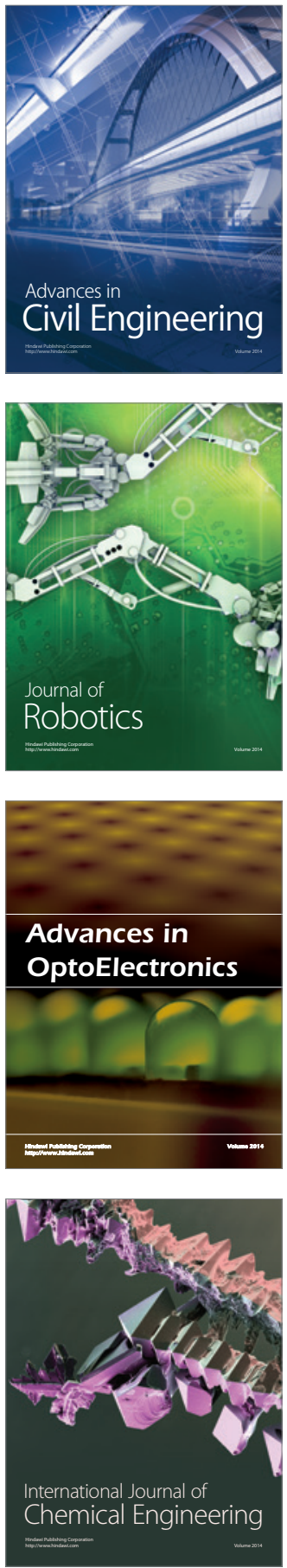

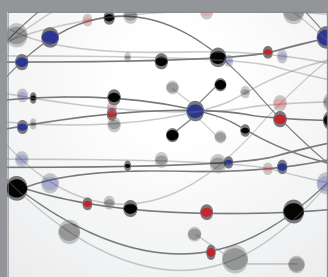

The Scientific World Journal

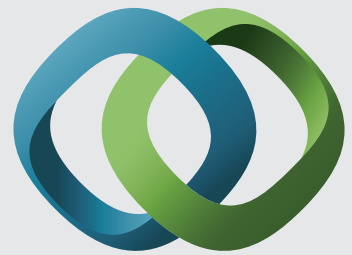

\section{Hindawi}

Submit your manuscripts at

http://www.hindawi.com
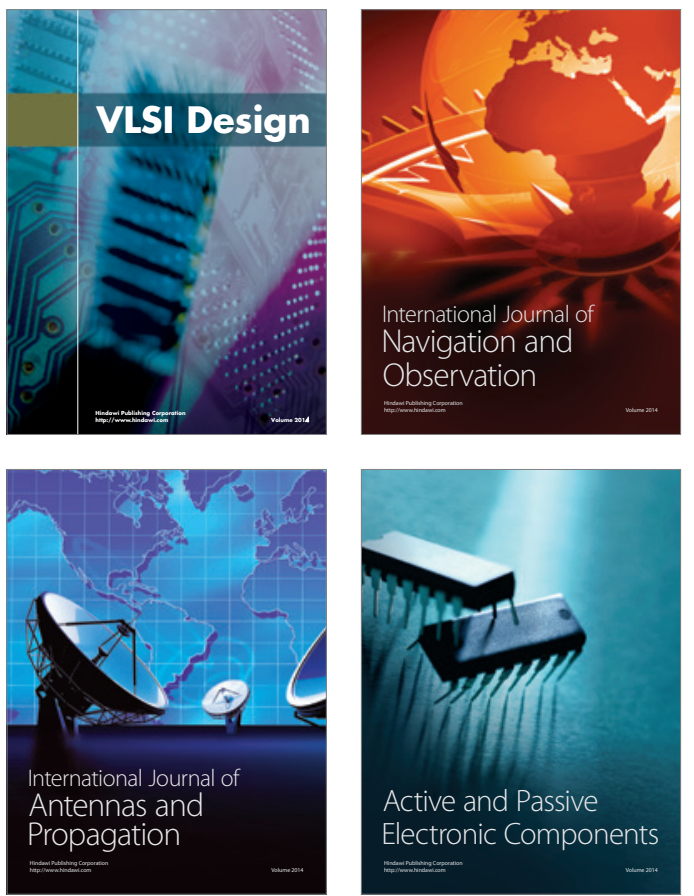
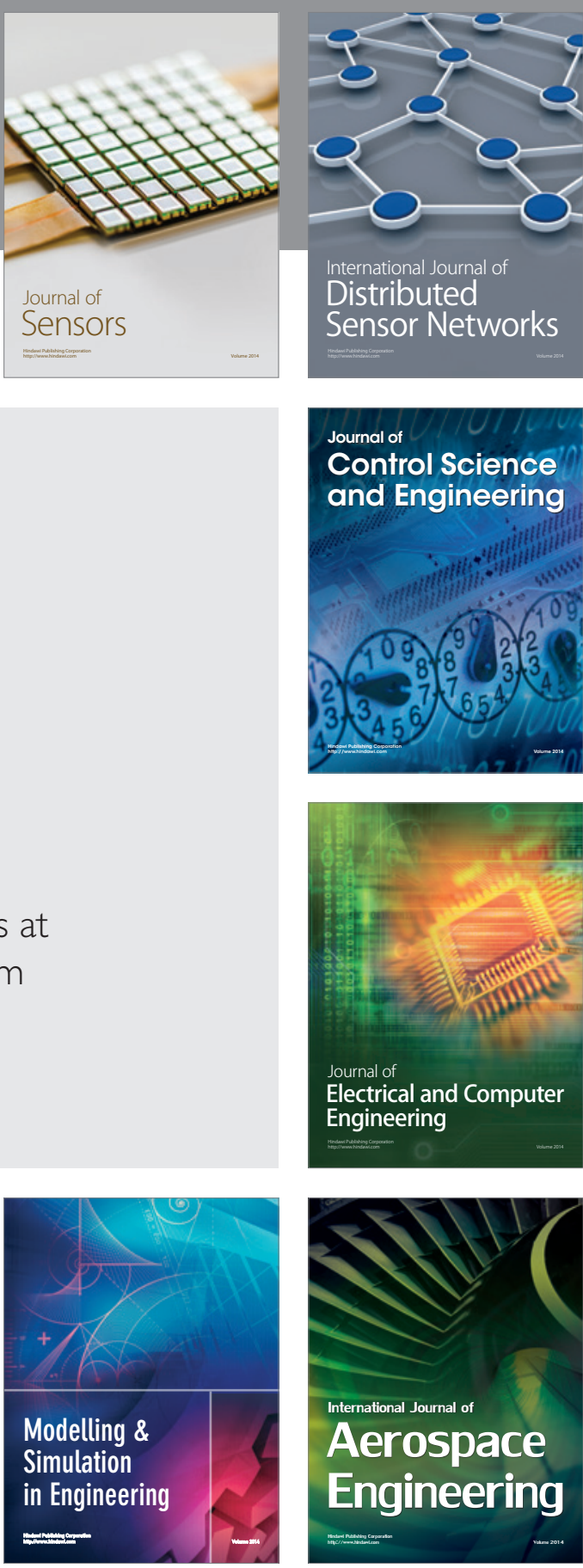

International Journal of

Distributed

Sensor Networks

Journal of

Control Science

and Engineering
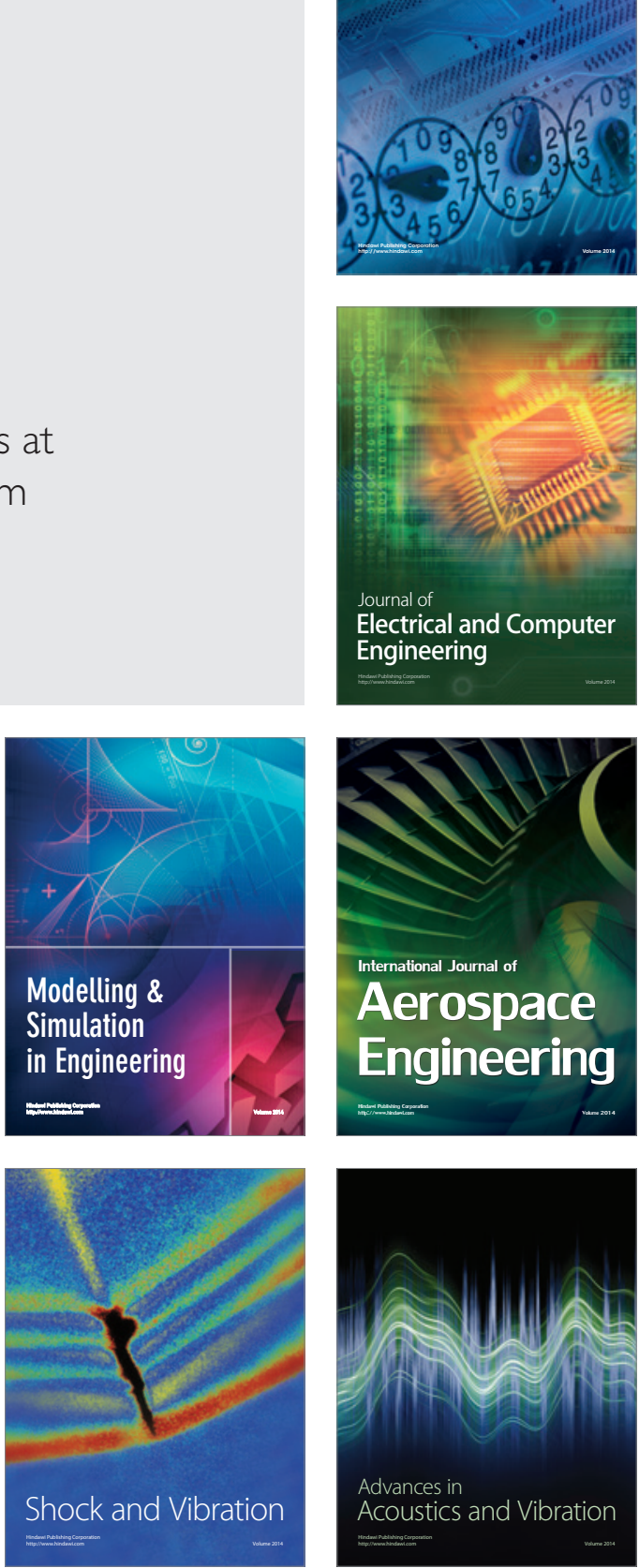